\title{
An Innovative Drive for Wheeled Mobile Robots
}

\author{
Jorge Angeles, Senior Member, IEEE
}

\begin{abstract}
Introduced in this paper is an innovative drive for wheeled mobile robots, that is based on two identical, coaxial wheels, independently driven by two identical motors. The common axis is capable of rotating about a vertical axis. The drive, termed Dual-Wheel Transmission (DWT), is composed of two identical epicyclic gear trains, lying at two different levels and coupled by a common planet-carrier. The latter can turn freely with respect to the robot platform carrying the motors, the transmission having, as a stand-alone unit, three degrees of freedom and only two motors, which makes it underactuated. Upon coupling this drive with two other wheel units, which is the minimum required for static support, a robot with mobility of three is produced, the underactuation thereby disappearing. Finally, the dimensioning of the DWT is reported for robustness against manufacturing, actuation and sensing errors.
\end{abstract}

Index Terms - Epicyclic gear trains, dual-wheel transmission, wheeled mobile robots.

\section{INTRODUCTION}

$\mathbf{T}$ RANSMISSION mechanisms for rolling vehicles under unmanned operation have been developed since the early automatic guided vehicles (AGV). Mechanism morphology varies, depending on the class of wheels, which can be conventional or omnidirectional. A conventional wheel is one with a simple disk geometry, omnidirectional wheels being of various types, notably Mekanum wheels, as disclosed by Fuchs [1], consisting of a drum on whose periphery a plurality of idle rollers are located, with axes skewed with respect to the axis of the drum. An alternative class of omnidirectional wheels comprises ball wheels, as disclosed by West [2], by Pin [3], and by Wada [4]. The transmission reported here is intended for conventional wheels.

Transmissions for conventional wheels can be of two kinds, depending on whether the axis of the wheel is fixed or articulated to the vehicle chassis. Fixed-axis wheels are restricted to vehicles with a mobility of two, identical to the mobility of manned terrestrial vehicles. Articulated wheels allow for a mobility of three, or full mobility, which allows, in turn, for the driving of a platform arbitrarily on a flat floor, with two independent translations, in the $x$ - and $y$-directions, plus a rotation in the plane. The transmission reported here pertains to the class of articulated wheels. The latter can be either idle or actuated; idle wheels require an offset between the steering and the driving axes, their actuated counterparts requiring two motors for their actuation, one for the steering and one for the driving. Moreover, articulated wheels can be either centered or offset, depending on whether the steering and the driving axes intersect or not. Offset wheels are termed caster (or castor) wheels. Our transmission pertains to centered wheels, but the concept can be equally applied to offset wheels.

J. Angeles is with Department of Mechanical Engineering McGill University, Montreal, Quebec, H3A 2K6 Canada (email: angeles@cim.mcgill.ca)
Caster wheels are common in applications not requiring an actuated wheel, such as in trolleys, the offset providing for a turning moment that allows the suitable reorientation of the wheel axis, as needed for pure rolling. Applications requiring the actuation of the wheel permit the use of centered wheels, provided that the wheel axis is steerable by means of a motor.

Caster wheels are common in the art. We can cite here the drive disclosed by Wienkop [5], with a wheel of the offset type, further developed by Legrand, Holmberg, and Slater [6]. A modification of this layout was proposed by Wada [4]. The foregoing patents disclose an actuation unit with one single wheel, which is both steerable and drivable by means of two identical motors of vertical axes. The steering and driving motions are transmitted to the wheel with the aid of one mechanism involving bevel gears with straight teeth, intended for the driving of the wheel about a horizontal axis. Wienkop's as well as Legrand and Slater's wheel units have their axis of rolling offset with respect to the vertical axis of steering, while Wada's bears one additional offset, that of the wheel plane, from the same steering axis.

The above inventions exhibit a drawback: one of the two identical motors is dedicated to the steering, the other to the driving, thereby performing two quite disparate functions that require disparate control strategies, i.e., position control vs. velocity control; additionally, the bevel gear train is more expensive than one with spur or helicoidal gears, besides being noisy, unless expensive, spiral teeth are used.

An alternative layout comprises a two-wheel unit, with the two wheels mounted coaxially, but turning at independent rates. A system like this is found in Carnegie-Mellon University's Pluto, developed by Moravec [7], [8]. Pluto is a wheeled robot with three actuation units, each supplied with two identical motors to both steer the common axis of the wheels and drive one of the two wheels, the second wheel being idle. This transmission is essentially different from those of Wienkop's, Legrand and Slater's and Wada's in that the motors with their speed reducers do not drive their assigned axes directly, but via a differential mechanism, which is made of bevel gears. This transmission exhibits its own drawbacks: a) the two identical motors are intended for two disparate functions, steering and driving; b) bevel gears entail the shortcomings mentioned above; and c) the driving of one single wheel limits the load-carrying capacity of the unit to one-half that provided by the two motors.

One further alternative layout, developed at Nanyang Technological University, Singapore, comprises a two-wheel unit as well, but with each of its two identical motors driving each of the two wheels with its own transmission [9]. The latter is intended to convert a rotation about the vertical axis of the motor into a rotation about the horizontal axis of symmetry of the wheels. This conversion is implemented by means of 
a worm-gear transmission, which works under friction, and hence, impacts on the efficiency of the device.

Below we describe an innovative drive, the Dual-Wheel Transmission (DWT), derive its kinematic relations, and provide guidelines for its robust dimensioning.

\section{DESCRIPTION OF THE DUAL-WHEEL TRANSMISSION}

Shown in Fig. 1 is the layout of the mechanism, consisting of the elements described below:

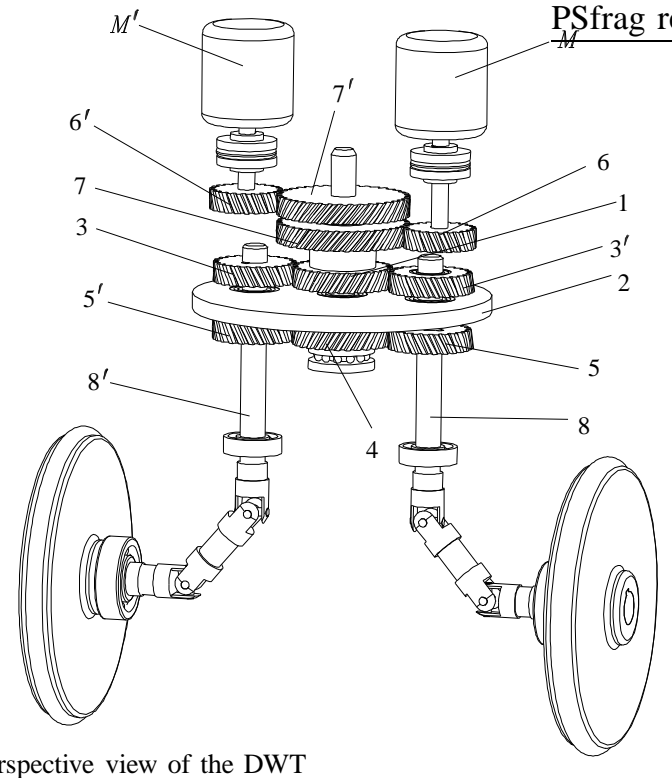

Fig. 1. Perspective view of the DWT

- The two motors $M$ and $M^{\prime}$ are rigidly mounted on the robot platform, not shown in the figure;

- the motors drive two epicyclic gear trains, at different levels, and composed of sun gears 1 and 4, respectively, these gears meshing with their corresponding planets 3 and $3^{\prime}$ for the former, 5 and $5^{\prime}$ for the latter;

- the two epicyclic trains are coupled by means of the common planet-carrier 2, which turns freely with respect to the robot platform;

- shaft $8^{\prime}$ is rigidly mounted on planet gear 3 and by means of bearings on planet gear $5^{\prime}$, while shaft 8 is rigidly mounted on planet gear 5 and by means of bearings on the planet gear $3^{\prime}$, as displayed in Figs. 2 and 3;

- shafts 8 and $8^{\prime}$ drive the two wheels by means of arrays of universal joints at $45^{\circ}$. These are preferred over more commonly used bevel gears or worm-gears because the former function under friction, besides entailing backlash, the latter being highly inefficient. Bevel gears with spiral teeth can function (almost) under pure rolling, but their high cost makes them rather unattractive in the art.

Notice that planets $3^{\prime}$ and $5^{\prime}$ are not kinematically essential, but are suggested here with the purpose of providing additional stiffness to the mounting of shafts 8 and $8^{\prime}$. Figure 4 illustrates the layout of Fig. 1 in front view for better visualization. In that figure, the point contact of the wheels with the ground is stressed, for this enhances the accuracy of the motions of the wheel unit and, hence, of the robot. In applications where accurate maneuvering is not a priority, contact at a large area

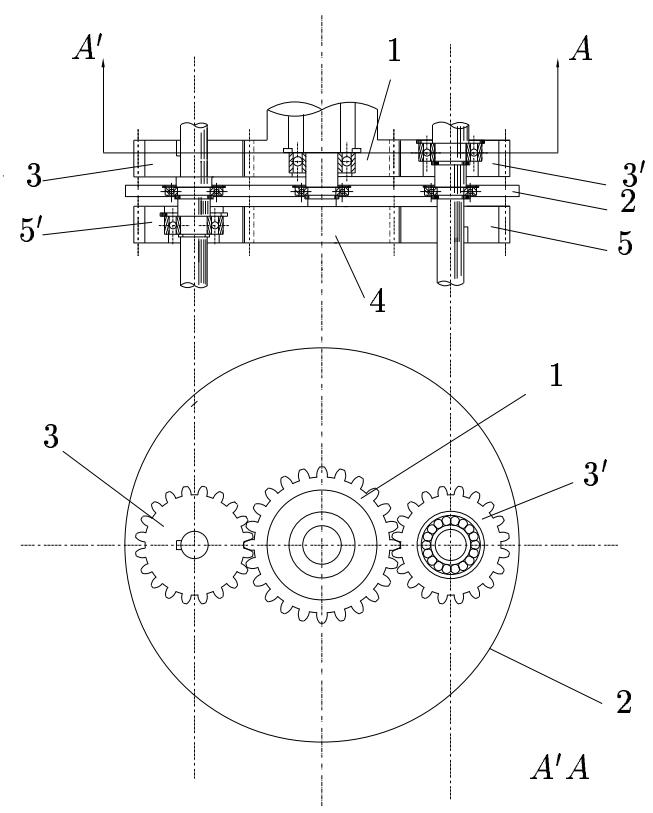

Fig. 2. Front view of epicyclic trains with section of upper train

can be tolerated, as is the case when pneumatic tires are used. Also note that $P$ is the intersection of the common axis of the sun gears with the common wheel axis, while $r$ denotes the radius of the wheels and $l$ the distance between the wheelground contact points. A prototype of the transmission was built by Leow Yong Peng, then a M.Eng. student at Singapore's Nanyang Technological University [10]. A photograph of the prototype is included in Fig. 5. Performance tests of the prototype will be reported in a forthcoming paper.

\section{DWT KINEMATICS}

The relations among the angular velocities of all moving elements are described herein, with the aid of the notation below ${ }^{1}$ :

$N_{i}$ : number of teeth of gear number $i$, for $i=1,3,4,5,6,7$, with the provision that any gear numbered with a prime has as many teeth as its unprimed counterpart;

$n_{13}=N_{1} / N_{3}$, the gear ratio between gears 1 and 3;

$n_{45}=N_{4} / N_{5}$, the gear ratio between gears 4 and 5;

$n_{61}=N_{6} / N_{1}$, the gear ratio between gears 6 and 1 ;

$\omega_{i}$ : angular velocity of the $i$ th rotating element, namely, gear, platform 0 , or planet-carrying disk 2 ;

$\omega_{i 0}$ : angular velocity of the $i$ th rotating element with respect to the robot chassis 0 ;

$\omega_{i 2}$ : angular velocity of the $i$ th rotating element with respect to the planet-carrier 2

$l$ : distance between the wheel-ground contact points.

$r$ : radius of the wheels;

$\rho=r / l$ : ratio of the wheel radius to the distance between contact points.

Although not necessary, it is highly advisable that the gear ratios of the two planetary trains be identical. Space concerns

\footnotetext{
${ }^{1} \omega_{i}, \omega_{i 0}$, and $\omega_{i 2}$ are defi ned for $i=0,1,2,3,3,4,5,5^{\prime}, 6,6^{\prime}, 7,7^{\prime}$.
} 


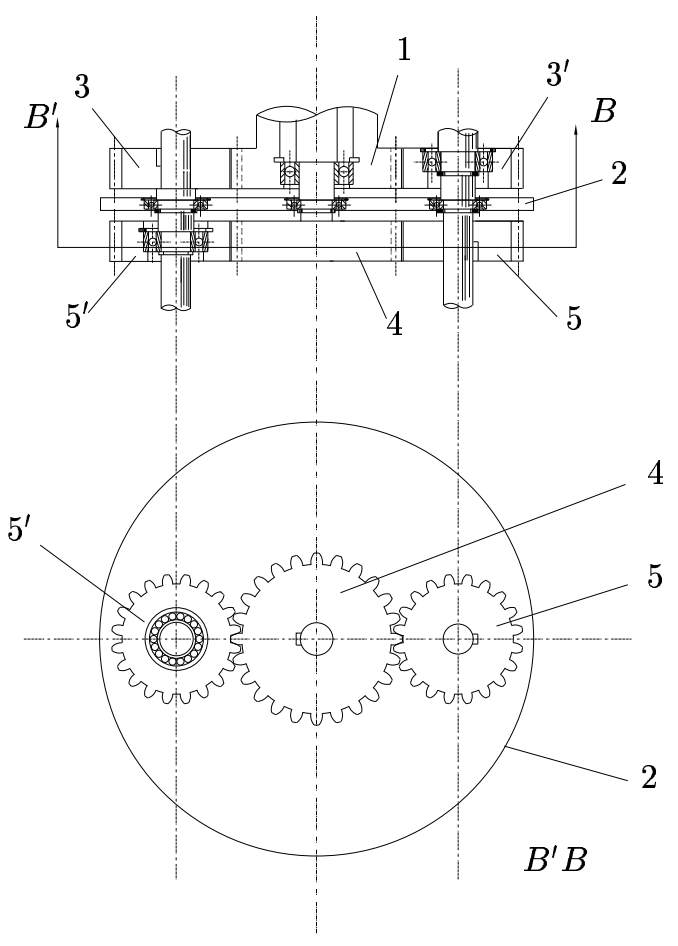

Fig. 3. Same as Fig.2, with section of lower train

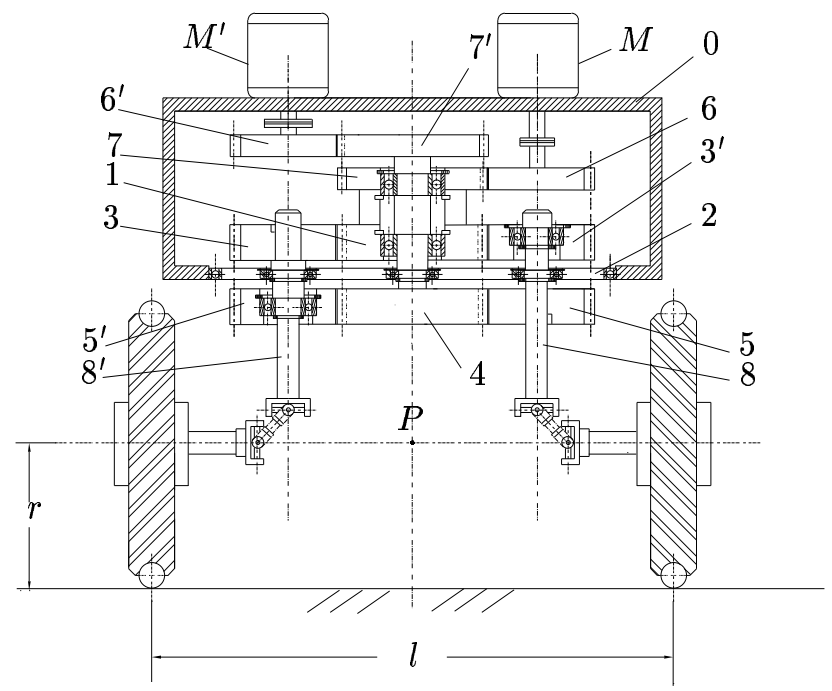

Fig. 4. Front view of the DWT unit

and equal gear ratios require that the pitch of gears 4,5 and $5^{\prime}$ be the same as that of gears 1,3 and $3^{\prime}$, and that the number of teeth in gear 1 be equal to that of gear 7. If this is the case, then we have the relations:

$$
n_{13}=n_{45}, \quad n_{67} \equiv \frac{N_{6}}{N_{7}}=n_{61}
$$

Moreover, we denote the velocity of $P$ by $v$.

From the kinematics of epicyclic gear trains [11], the relations among the velocities of the two trains are readily derived as

$$
\omega_{12}=\omega_{0}-\omega_{2}-n_{61} \omega_{60}, \quad \omega_{42}=\omega_{0}-\omega_{2}-n_{61} \omega_{6^{\prime} 0}
$$

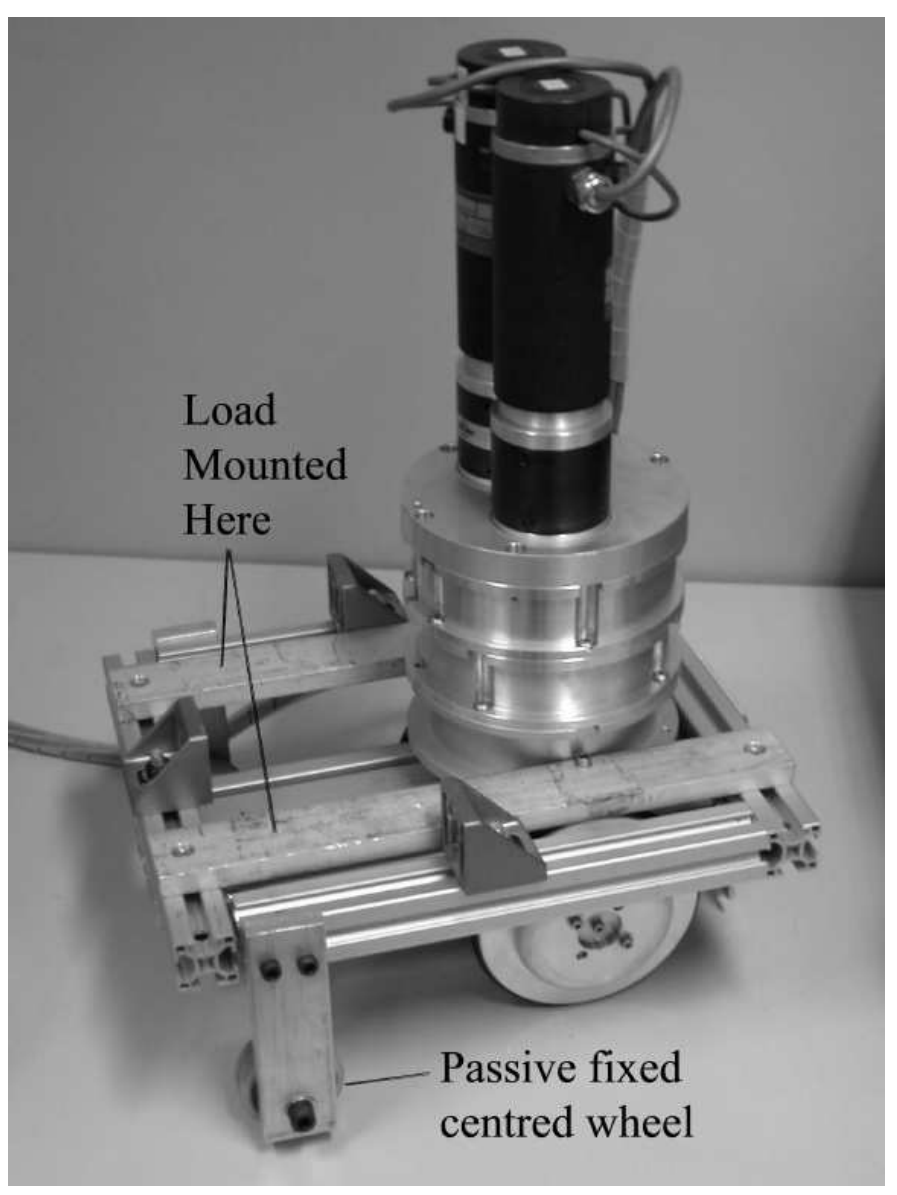

Fig. 5. A photograph of the prototype

Moreover, if we assume further that $n_{13}=1 / n_{61}$, then

$$
\omega_{12}=-n_{61} \omega_{32}, \quad \omega_{42}=-n_{61} \omega_{52}
$$

Substitution of eqs.(3) into eqs.(2) yields, in terms of velocities with respect to the platform,

$$
\begin{aligned}
& \omega_{32}=\omega_{60}+\frac{1}{n_{61}}\left(\omega_{2}-\omega_{0}\right) \\
& \omega_{52}=\omega_{6^{\prime} 0}+\frac{1}{n_{61}}\left(\omega_{2}-\omega_{0}\right)
\end{aligned}
$$

Further, note that the horizontal components of the absolute angular velocities of the left and right wheels of Fig. 4 are identical to the relative angular velocities $\omega_{32}$ and $\omega_{52}$ of the two shafts driving the wheels. Under the assumption that the two wheels roll without slipping and without skidding, these velocities are related to that of the planet-carrier, $\omega_{2}$, by

$$
\omega_{2}=\rho\left(\omega_{32}+\omega_{52}\right)
$$

where the positive sign accounts for the reversal in the direction of the angular velocity caused by the bends in opposite directions provided by the pair of universal joints. Likewise, the velocity of $P$ is related to the angular velocities of the driving shafts by

$$
v=\frac{r}{2}\left(\omega_{32}-\omega_{52}\right)
$$


Notice that, while eq.(5b) displays a relation between $v$ and the difference in shaft angular velocities with respect to the planet-carrier 2, the relation would be equally valid with absolute angular velocities. The reason is that the common angular velocity $\omega_{0}+\omega_{2}$ required to produce absolute angular velocities from the ones displayed would be canceled by the difference. However, in eq.(5a), it is imperative to use relative angular velocities with respect to the planet-carrier.

\section{A. Forward Kinematics}

We define here the forward-kinematics (FK) relations of the DWT unit as those expressing explicitly the Cartesian velocities of the unit in terms of the actuator variables. Now, the Cartesian velocities, for one single DWT unit, are two: the angular velocity $\omega_{2}$ of the planet-carrier-i.e., the angular velocity of the common wheel axis-and the velocity $v$ of point $P$. The actuator variables are the angular velocities $\omega_{60}$ and $\omega_{6^{\prime}} 0$ produced by the motors. The FK relations are derived below.

For a given pair of angular velocities $\omega_{60}$ and $\omega_{6^{\prime} 0}$, along with the prescribed angular velocity $\omega_{0}$ of the platform, we can obtain the corresponding angular velocity $\omega_{2}$ of the planet-carrier and the velocity $v$ of the midpoint $P$ of the wheel common axis. To this end, we substitute eqs.(4a \& b) into eqs. $(5 \mathrm{a} \& \mathrm{~b})$. After simple rearrangements, the desired expressions are

$$
\begin{aligned}
\omega_{2} & =\frac{n_{61} \rho}{n_{61}-2 \rho}\left(\omega_{60}+\omega_{6^{\prime} 0}-\frac{2}{n_{61}} \omega_{0}\right) \\
\frac{v}{r} & =\frac{1}{2}\left(\omega_{60}-\omega_{6^{\prime} 0}\right)
\end{aligned}
$$

Moreover, eqs.(4a \& b) are now solved for $\omega_{0}$, thereby obtaining two different values for this variable, namely,

$$
\begin{aligned}
& \omega_{0}=\omega_{2}+n_{61}\left(\omega_{60}-\omega_{32}\right) \\
& \omega_{0}=\omega_{2}+n_{61}\left(\omega_{6^{\prime} 0}-\omega_{52}\right)
\end{aligned}
$$

The two foregoing expressions are now combined into a single one by adding sidewise these expressions:

$$
\omega_{0}=\omega_{2}+\frac{1}{2} n_{61}\left[\omega_{60}+\omega_{6^{\prime} 0}-\left(\omega_{32}+\omega_{52}\right)\right]
$$

Recalling eq.(5a), the above expression for $\omega_{0}$ can be expressed free of $\omega_{2}$, namely,

$$
\omega_{0}=\left(\rho-\frac{n_{61}}{2}\right)\left(\omega_{32}+\omega_{52}\right)+\frac{n_{61}}{2}\left(\omega_{60}+\omega_{6^{\prime} 0}\right)
$$

Equations $(6 a-c)$ are the FK relations sought. Notice that these relations show that the DWT unit is underactuated. Underactuation disappeares when this unit is coupled with other units either of the same kind or of other kinds of wheels, to form a wheeled robot.

\section{B. Inverse Kinematics}

Regarding the control of the DWT unit, a maneuver of the robot is specified by the angular velocity $\omega_{0}$ of the platform and the velocity $v$ of the midpoint $P$. The angular velocity of the planet-carrier $\omega_{2}$, moreover, is specified by the trajectory to be tracked, as this is the steering velocity of the wheel unit.
With these data, the required relative angular velocities of the two motors with respect to the platform are to be computed. These are readily obtained from the FK relations, derived from eqs.(6a \& b), namely,

$$
\begin{aligned}
\omega_{60} & =\frac{1}{n_{61}} \omega_{0}+\frac{n_{61}-2 \rho}{2 \rho n_{61}} \omega_{2}+\frac{v}{r} \\
\omega_{6^{\prime} 0} & =\frac{1}{n_{61}} \omega_{0}+\frac{n_{61}-2 \rho}{2 \rho n_{61}} \omega_{2}-\frac{v}{r}
\end{aligned}
$$

While not all three Cartesian variables can be controlled with the two motors of the unit, it is possible to control them when the unit is used to drive a mobile platform, as explained above.

\section{Platform-Velocity Sensing}

The effective control of the robot motion calls for platformmotion-sensing, which is possible with internal sensors, i.e., on-board velocity sensors. These can be collocated at the mountings of the shafts with the planet-carrier, thereby producing readouts of $\omega_{32}$ and $\omega_{52}$. Using these readouts, along with those of $\omega_{60}$ and $\omega_{6}{ }^{\prime}$, provided by the motor encoders, the planet-carrier angular velocity $\omega_{2}$ and the velocity $v$ of the midpoint $P$ can be inferred from eqs. $(6 \mathrm{a} \& \mathrm{~b})$. Moreover, recalling eq.(5a), $\omega_{0}$, as given by eq.(6c), is expressed free of $\omega_{2}$, thereby obtaining an enhanced estimate of the platform absolute angular velocity $\omega_{0}$ using four on-board encoders whose readouts are, moreover, averaged. The velocity of a landmark point of the platform requires, in turn, an estimate of the velocity $v$ of $P$, which is given by eq.(6b).

The data transmission of the measurement of angular velocities $\omega_{32}$ and $\omega_{52}$ requires the use of wireless technology. Hardwiring these sensors to the processing unit will re-introduce the problem of wire entangling and hamper an important benefit of the DWT.

As an alternative, the angular velocity $\omega_{20}$ of the planetcarrier 2 with respect to the platform 0 may be sensed without this problem. To this end, we introduce $\omega_{20}=\omega_{2}-\omega_{0}$ into eqs.(4a \& b), substitute the result into eq.(5a) and solve for $\omega_{0}$, thus obtaining

$$
\omega_{0}=\left(\frac{2 \rho}{n_{61}}-1\right) \omega_{20}+\rho\left(\omega_{60}+\omega_{6^{\prime} 0}\right)
$$

Finally, note that the measurement of $\omega_{20}$ is redundant when two or more DWTs are coupled, in which case the DWT loses underactuation.

\section{The Robust Design of The DWT Unit}

The key design variables are $n_{61}$ and $\rho$. The prototype displayed in Fig. 5 was dimensioned with $n_{61}=0.8$ and $\rho=0.47$, values which were decided upon by Mr. Leow [10] using engineering judgment, in the absence of sharp design quidelines. We report in this section on optimum, practical values of these variables, found with the purpose of adding robustness to the design.

Robustness was proposed by Taguchi [12] in the postwar years as a means to make Japanese products of the times more competitive with their western counterparts. While robust engineering is broadly accepted by industry as a means to 
improve the quality of goods and services, the concept has not as yet been applied in design engineering to its fullest extent. The reason may lie in the lack of a theoretical framework. We proposed recently such a framework, whereby robustness is measured in terms of the condition number of what we term the design-performance matrix [13], [14], namely, the matrix mapping variations in the design-environment parameters $(D E P)$ - the parameters occurring in the mathematical model of a design and over which the designer has no controlinto variations in the design-performance functions. The latter relate the performance variables with the DEP and the design variables, which are those to be determined by the designer.

Resorting to the robust-design formulation recalled above, we consider the performance of the DWT under both kinematic control and velocity sensing.

Kinematic control pertains to the production of the velocity variables associated with a maneuver, namely, $\omega_{0}, \omega_{2}$ and $v / r-v$ is divided by $r$ in order to produce velocity variables with the same units-by means of the actuator joint rates $\omega_{60}$ and $\omega_{6^{\prime}}$. The pertinent relations are better visualized upon casting eqs. (7a \& b) in vector form, which yields

$$
\mathbf{A t}=\dot{\boldsymbol{\theta}}_{a}
$$

where we have introduced the vector of actuation rates $\dot{\boldsymbol{\theta}}_{a}$, the vector of controlled-velocity variables $\mathbf{t}-$ we use the symbol t to represent the controlled variables, which, in robotics, are normally grouped into an array of end-effector or robotplatform variables defining the velocity field in the body and termed twist - and the forward-kinematics Jacobian A, or actuation matrix, all these items defined as

$$
\begin{aligned}
& \mathbf{A} \equiv \frac{1}{n_{61}}\left[\begin{array}{ccc}
1 & \left(n_{61}-2 \rho\right) /(2 \rho) & n_{61} \\
1 & \left(n_{61}-2 \rho\right) /(2 \rho) & -n_{61}
\end{array}\right], \\
& \dot{\boldsymbol{\theta}}_{a} \equiv\left[\begin{array}{c}
\omega_{60} \\
\omega_{6 / 0}
\end{array}\right], \quad \mathbf{t} \equiv\left[\begin{array}{c}
\omega_{0} \\
\omega_{2} \\
v / r
\end{array}\right]
\end{aligned}
$$

Apparently, the designer has no control over which maneuver, represented by $\mathbf{t}$, the robot will be commanded to execute, and hence, $\mathbf{t}$ constitutes the DEP vector, $\dot{\boldsymbol{\theta}}_{a}$ being the vector of performance functions. The design variables, in turn, are $\rho$ and $n_{61}$.

In computing the inverse relations of eq.(9a), matrix A plays an important role in that, if ill-conditioned, then the results of the inverse relations will be corrupted with an inadmissibly large roundoff-error amplification. In this vein, it is important to design the DWT so as to keep the condition number $\kappa$ of A as small as possible. The condition number of any matrix is bounded by unity from below and unbounded from above. We thus aim at minimizing the condition number $\kappa(\mathbf{A})$. When the Frobenius norm [15] is used to define $\kappa(\mathbf{A})$, we have the relations

$$
\kappa(\mathbf{A})=\sqrt{\kappa\left(\mathbf{A} \mathbf{A}^{T}\right)}
$$

and

$$
\kappa\left(\mathbf{A} \mathbf{A}^{T}\right)=\left\|\mathbf{A} \mathbf{A}^{T}\right\|\left\|\left(\mathbf{A} \mathbf{A}^{T}\right)^{-1}\right\|
$$

with $\left\|\mathbf{A} \mathbf{A}^{T}\right\|$ indicating the Frobenius norm of $\mathbf{A} \mathbf{A}^{T}$, namely,

$$
\left\|\mathbf{A} \mathbf{A}^{T}\right\|=\sqrt{\operatorname{tr}\left[\frac{1}{n}\left(\mathbf{A A}^{T}\right)^{2}\right]}
$$

and $\operatorname{tr}(\cdot)$ indicating the trace of $(\cdot)$ for any $n \times n$ matrix $\mathbf{A} \mathbf{A}^{T}$, where $n=2$ in our case; a similar definition follows for $\left\|\left(\mathbf{A A}^{T}\right)^{-1}\right\|$. After some simple but long calculations, which are implemented using computer algebra, we obtain

$$
\kappa(\mathbf{A})=\frac{1+\left(n_{61} / 2 \rho-1\right)^{2}+n_{61}^{2}}{2 n_{61} \sqrt{1+\left(n_{61} / 2 \rho-1\right)^{2}}}
$$

As the reader can readily verify, it is possible to make $\kappa(\mathbf{A})=1$ upon choosing the design variables $\rho$ and $n_{61}$ as $1 / 2$ and unity, respectively. A matrix with a condition number of unity has all its singular values identical and nonzero; such a matrix is termed isotropic. However, as the reader can also verify upon looking at eqs.(7a \& b), this choice would lead to an uncoupling of the angular velocity $\omega_{2}$ of the planet-carrier from the angular velocities $\omega_{60}$ and $\omega_{6^{\prime} 0}$, thereby making a change of course impossible ${ }^{2}$. We thus avoid this choice, and look for alternative values of the design variables that render $\kappa(\mathbf{A})$ a minimum.

The normality conditions for a stationary value-minimum, maximum, or saddle point - of $\kappa^{2}(\mathbf{A})$ are readily derived with the aid of computer algebra as well. These conditions lead to

$$
\begin{aligned}
& f_{1}\left(n_{61}, \rho\right)=\left(\frac{n_{61}}{2 \rho}-1\right) F G=0 \\
& f_{2}\left(n_{61}, \rho\right)=F G=0
\end{aligned}
$$

with $F$ and $G$ defined as

$$
\begin{aligned}
& F\left(n_{61}, \rho\right)=1+n_{61}^{2}+\left(\frac{n_{61}}{2 \rho}-1\right)^{2} \\
& G\left(n_{61}, \rho\right)=1-n_{61}^{2}+\left(\frac{n_{61}}{2 \rho}-1\right)^{2}
\end{aligned}
$$

Apparently, $F>0$, and hence, the only possible relation between the two design variables, under which eqs.(12a \& b) vanish simultaneously is $G=0$, i.e.,

$$
1-n_{61}^{2}+\left(\frac{n_{61}}{2 \rho}-1\right)^{2}=0
$$

which defines an optimum locus of values of the two design variables $n_{61}$ and $\rho$. We shall refer to this locus as contour 1 . Any pair of design variables lying on contour 1 yields the same minimum value of $\kappa(\mathbf{A})$.

With regard to velocity sensing, the pertinent relations are those mapping the measured variables, $\omega_{32}, \omega_{52}, \omega_{60}$ and $\omega_{6^{\prime} 0}$ into the controlled variables $\omega_{0}$ and $v / r$. These relations stem from eqs.(5b) and (6c); in vector form, these relations become

$$
\mathbf{S y}=\omega
$$

\footnotetext{
${ }^{2}$ Such uncoupling is at the core of the functioning of the South Pointing Chariot mechanism [16]
} 
with $\mathbf{y}, \boldsymbol{\omega}$ and $\mathbf{S}$ defined as

$$
\begin{array}{r}
\mathbf{y} \equiv\left[\begin{array}{c}
\omega_{32} \\
\omega_{52} \\
2 \bar{\omega}
\end{array}\right], \quad \boldsymbol{\omega} \equiv\left[\begin{array}{c}
\omega_{0} \\
v / r
\end{array}\right], \\
\mathbf{S} \equiv\left[\begin{array}{ccc}
\rho-n_{61} / 2 & \rho-n_{61} / 2 & n_{61} / 2 \\
1 / 2 & -1 / 2 & 0
\end{array}\right]
\end{array}
$$

where $\bar{\omega}$ is the mean value of the measurements of the two angular velocities $\omega_{60}$ and $\omega_{6 / 0}$, i.e.,

$$
\bar{\omega} \equiv \frac{1}{2}\left(\omega_{60}+\omega_{6 / 0}\right)
$$

It should be clear now that $\mathbf{y}$ comprises the DEP, while $\boldsymbol{\omega}$ the performance functions, the design variables being, again, $n_{61}$ and $\rho$. Designing for robustness is done by minimizing the condition number of the sensing matrix $\mathbf{S}$, which leads us to the minimization of $\kappa(\mathbf{S})$. Moreover, since the condition number of $\mathbf{S}$ based on the Frobenius norm is the square of that of $\mathbf{S S}^{T}$, we calculate this product below:

$$
\mathbf{S S}^{T}=\left[\begin{array}{cc}
2\left(\rho-n_{61} / 2\right)^{2}+n_{61}^{2} / 4 & 0 \\
0 & 1 / 2
\end{array}\right]
$$

and hence, $\mathbf{S S}^{T}$ is a $2 \times 2$ diagonal matrix, which can be rendered isotropic, rather simply. The isotropy condition of $\mathbf{S S}^{T}$, and hence, of $\mathbf{S}$, reduces, then, to forcing the two diagonal entries of $\mathbf{S S}^{T}$ to be identical, i.e.,

$$
H \equiv 2 \rho^{2}-2 \rho n_{61}+\frac{3}{4} n_{61}^{2}-\frac{1}{2}=0
$$

thereby obtaining what we shall term contour 2 in the $n_{61}-\rho$ plane. We now have two contours in this plane, as displayed in Fig. 6, their intersections yielding the two real, positive solutions that minimize the condition numbers of the two matrices $\mathbf{A}$ and $\mathbf{S}$ simultaneously ${ }^{3}$. By inspection, we obtain

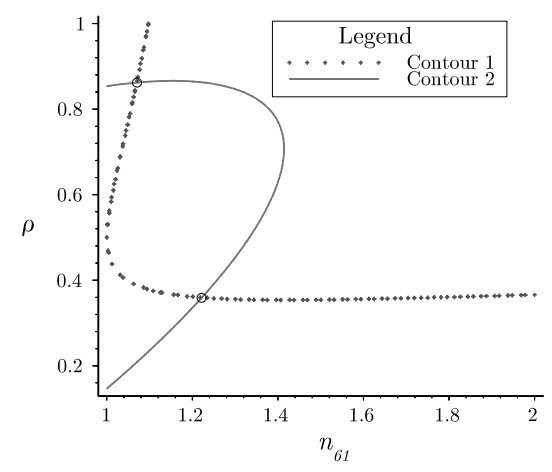

Fig. 6. The two minimum-condition-number loci in the $n_{61}-\rho$ plane

two pairs of real, positive values, namely, $(1.224,0.380)$ and $(1.072,0.860)$. We thus have two possible choices. Since $r$ should be substantially smaller than $l, \rho$ should be likewise smaller than 1 , the obvious choice thus being the first pair of

\footnotetext{
${ }^{3}$ Actually, full plots yield four intersections, the other two being symmetrically located with respect to the origin, and hence, yield negative values of the design variables.
}

values. However, $n_{61}$ being the ratio of two tooth numbers, 1.22 is not practical. A more practical value would be $n_{61}=$ 1.25 , which can be realized with reasonable numbers of teeth, namely, $N_{1}=12$ and $N_{6}=15$. Now, if we choose this slightly modified value of $n_{61}$, the above value of $\rho$ is no longer in any of the optimum loci of Fig. 6. An adjustment is warranted, which can be done by substitutiing the foregoing value of $n_{61}$ into both eq.(13) and eq.(15). In each case we obtain a different value of $\rho$. For the former, we obtain, with only four decimals displayed, $\rho_{11}=2.5000, \quad \rho_{21}=0.3571$, while, for the latter, $\rho_{12}=0.8589, \quad \rho_{22}=0.3911$. Obviously, our best choice is the mean value of the two above values closest to the original value of 0.380 , i.e.,

$$
\rho_{\text {opt }}=\frac{1}{2}\left(\rho_{21}+\rho_{22}\right)=0.3741
$$

the obvious choice for a practical design being 0.3750 , i.e.,

$$
\rho=\frac{r}{l}=0.3750 \Rightarrow r=\frac{3}{8} l
$$

the optimum diameter of the wheels, for a robust design, thus being $75 \%$ of the distance between wheels. As a matter of fact, the Frobenius-norm-based condition number of matrix $\mathbf{S}$ turns out to be, for the two chosen values of $n_{61}$ and $\rho$, $\kappa(\mathbf{A})=1.0008$, with five decimals, which is reasonable close to the lower bound of unity. Likewise, $\kappa(\mathbf{S})$ turns out to be $\kappa(\mathbf{S})=1.0155$, with five decimals, and hence, a fairly low value as well.

\section{Practical Aspects of the Design}

The design proposed here offers interesting features for a robust performance:

- The averaging effect of the sensing allowed by the symmetry of the array, as made apparent by eqs. $6 \mathrm{~b} \&$ c), provides an enhancement of the encoder resolution, besides providing for error-filtering of the sensed signals.

- The indirect driving of the wheel shafts eliminates problems of wire entanglement, while allowing for unlimited rotation possibilities of the common horizontal axis of the two wheels.

- The actuation of the two wheels allows for a total use of the power supplied by the two motors, and hence, for an enhanced load-carrying capacity, besides providing for a uniform wear of the tires - not possible with other dualwheel units that provide actuation on one single wheel, while leaving the other wheel idle.

- The robust dimensioning of the key parameters of the unit, yielding a quasi isotropic design, renders the unit least sensitive to manufacturing, actuation, and sensing errors.

\section{Conclusions}

We introduced a novel drive for wheeled mobile robots that consists of two epicyclic gear trains lying at different levels. The drive, termed dual-wheel transmission, is actuated by two motors, but entails three velocity variables. This underactuation is eliminated upon mounting the unit on a robot platform that couples it with the other robot wheels. 
Design guidelines were given and optimum values of the key design variables were obtained by minimizing the condition numbers of the two matrices describing the kinematics of the unit. These are the actuation and the sensing matrices. The optimum values obtained were adjusted to allow for a practical design. After this adjustment, the condition numbers of the two matrices remained quite close to unity, which guarantees robustness against measurement and control-signal errors. It was made apparent that the symmetries involved lead to an enhancement of the sensor resolution, by providing relations between averaged variables.

\section{ACKNOWLEDGEMENTS}

The research and design work reported here were possible under NSERC (Canada's Natural Sciences and Engineering Research Council) Research Grant OGP0004532. Part of the work was conducted while the author was on sabbatical at Nanyang Technological University, of Singapore. The support of NTU's School of Mechanical and Production Engineering and the Singapore Institute of Manufacturing Technology (SIMTech), which sponsored the construction of a prototype, are duly acknowledged. The design and construction of the prototype was under the excellent care of Leow Yong Peng, a SIMTech engineer.

\section{REFERENCES}

[1] C. Fuchs, "Rad, das eine unmittelbare Fortbewegung in jeder Richtung gestattet," German Patent \# 822,660, Class 63d, Group 1, July 81949.

[2] A. M. T. West, "Omnidirectional vehicle," U.S. Patent \# 5,186,270, February 16, 1993.

[3] F. G. Pin and S. M. Killough, "Omni-directional and holonomic rolling platform with decoupled rotational and translational degrees of freedom," U. S. Patent \# 5,374,879, December 20, 1994.

[4] M. Wada, "Omnidirectional vehicle and method of controlling the same," U.S. Patent \# 6408230, June 18, 2002.

[5] U. Wienkop, "Robot hallway traveler," U.S. Patent \# 5,576,947, November 19, 1996.

[6] R. M. Legrand, R. Holmberg, and J. C. Slater, "Method and apparatus for mobile robot motion control," EP1037129, September 2000.

[7] H. Moravec, "Three degrees for a mobile robot," in ASME Conference on Computers in Engineering, Las Vegas, August 12-15, 1984.

[8] "The Stanford cart and the CMU rover," in Proceedings of the IEEE, July, 1983, pp. 872-884.

[9] G. Seet, R. S. Senayake, , and E. Low, "Autonomous Mobile Robot for Hospitals," Journal of Robotics and Mechatronics, vol. 7, no. 3, pp. 263-269, 1995.

[10] Y. Leow, "Kinematic modelling, mobility analysis and design of wheeled mobile robots," Master's thesis, Department of Mechanical Engineering, Nanyang Technological University, Singapore, 2002.

[11] R. L. Norton, Design of Machinery, 2nd ed. New York: McGraw-Hill, 2001.

[12] G. Taguchi, S. Chowdhury, and S. Taguchi, Robust Engineering. New York: McGraw Hill Book Co., 2000.

[13] K. Al-Widyan, J. Angeles, and J. Cervantes-Sánchez, "A model-based framework for robust design," in Gogu, G. et al. (eds.), Integrated Design and Manufacturing in Mechanical Engineering, Dordrecht, 2003.

[14] K. Al-Widyan and J. Angeles, "A model-based formulation of robust design," ASME Journal of Mechanical Design, 2004, accepted for publication.

[15] G. Golub and F. V. Loan, Matrix Computations. Baltimore, Maryland: The John Hopkins University Press, 1983.

[16] L.-C. Hsieh, J.-Y. Liu, and M.-H. Hsu, "Systematic method for the synthesis of South Pointing Chariot with planetary gear trains," Trans. Canadian Society for Mechanical Engineering, vol. 20, no. 4, pp. 421-435, 1996.
Jorge Angeles obtained his diploma in Electromechanical Engineering from the National University of Mexico (UNAM, 1969), a M.Eng. in Mechanical Engineering (1970) from UNAM as well, and a Ph.D. in Applied Mechanics (1973) from Stanford University. Between 1973 and 1984, Angeles held academic positions at UNAM. Since 1984, Angeles is with McGill University's Department of Mechanical Engineering, while holding an affi liation with McGill's Centre for Intelligent Machines.

Angeles has authored or co-authored various books in the areas of kinematics and dynamics of mechanical systems as well as numerous technical papers in refereed journals and conference proceedings. Angeles' research interests focus on the theoretical and computational aspects of multibody mechanical systems for purposes of design and control. Besides his research activities, Angeles is a consultant to various Canadian and

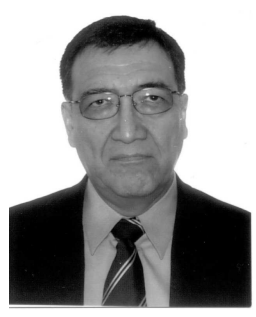
international organizations in matters of automation, mechanical design, and robotics; he also holds two patents, with other ones currently filed. Jorge Angeles is a Fellow of the Royal Society of Canada, a James McGill Professor of Mechanical Engineering at McGill University; a NSERC Design Engineering Chair holder; an ASME Fellow; a Fellow of the Canadian Society for Mechanical Engineering; a Honorary Member of IFToMM, the International Federation for the Promotion of Mechanism and Machine Science; a Senior Member of the IEEE; Past President of IFToMM; and a member of various professional and learned societies. Professional registration as an engineer includes Quebec, Mexico, and Germany. Angeles has held visiting positions at Aachen Institute of Technology (Germany), the Technical University of Munich (Germany) and Nanyang Technological University (Singapore). He was Nanyang Professor at Nanyang Technological University in 20002002 and is a Honorary Professor at Tianjin University, in China. 